\title{
L'aménagement hydroélectrique du territoire de la baie James
}

\author{
Hydro-electric development \\ of James bay
}

\author{
G. Marinier
}

Vice-président

Ingéniérie et Développement

Société d'énergie de la Baie James

Dix ans se sont écoulés depuis les débuts du projet de la Baie James. L'envergure des travaux à réaliser posait certes de nombreux défis quand a démarré le plus grand chantier du monde en 1971. Aujourd'hui, on peut presque affirmer que la mission est accomplie devant le bilan des réalisations de la première phase du Complexe hydroélectrique de la Grande Rivière. Mais il reste beaucoup à faire : encore près de vingt ans de travaux seront nécessaires pour achever la deuxième phase de ce complexe et mener à terme deux autres importants projets : le Complexe de la Grande rivière de la Baleine et le Complexe des rivières Nottaway, Broadback et de Rupert.

En fait, l'idée d'aménager les cours d'eau du territoire de la Baie James, dans le Moyen Nord québécois, remonte au début des années 60 . Déjà à cette époque, la société d'état Hydro-Québec se préoccupait du potentiel hydroélectrique aménagé qui, compte tenu du rythme de progression de la demande, allait vite devenir insuffisant. La centrale des chutes Churchill et les dernières centrales du Complexe Manicouagan-Outardes allaient permettre de répondre aux besoins des Québécois jusqu'à la fin des années 70, mais il fallait trouver de nouvelles ressources pour les années 80 . C'est à ces besoins que devait répondre le projet de la Baie James, désormais connu sous le nom de Complexe La Grande et divisé en deux phases : la Phase $\mathrm{I}$, présentement en cours, et la Phase II, à venir.

Alors que les projets antérieurs avaient été construits relativement près des grandes voies de communication, ceux du territoire de la Baie James allaient prendre forme dans un paysage quasi sibérien, à plus de $600 \mathrm{~km}$ au nord de Matagami, petite ville minière où s'arrêtait l'infrastructure routière et ferroviaire. Aux particularités du terrain, s'ajoutait la difficulté inhérante à l'envergure des travaux (Fig. 1).
C'est dans ce contexte inédit, mettant en jeu des investissements de plusieurs milliards de dollars, que le gouvernement du Québec créait, en 1971, la Société d'énergie de la Baie James à qui il confiait le mandat d'exploiter les ressources hydroélectriques de ce territoire.

Ce territoire couvre $176000 \mathrm{~km}^{2}$, soit près du tiers de la superficie totale de la France. Il s'étend sur une distance de $725 \mathrm{~km}$ de l'est à l'ouest, et de $745 \mathrm{~km}$ du nord au sud. Au plus fort des travaux, 18000 travailleurs allaient y braver l'isolement, la solitude et le froid pour entreprendre ce qui a été qualifié de "projet du siècle".

\section{Les débuts du "projets du siècle"}

En 1971, on a donc entrepris la construction d'une route de pénétration longue de $620 \mathrm{~km}$ pour relier Matagami au premier chantier du complexe, La Grande 2 , situé à $111 \mathrm{~km}$ de la baie James.

Cette route qui traverse une région hostile, où une multitude de rivières, de marécages et de baissières constituaient autant d'obstacles, a été construite en moins de deux ans au rythme de un kilomètre par jour. Asphaltée sur toute sa longueur et comportant dix ponts, elle devait permettre aux véhicules lourds de circuler en toutes saisons et d'acheminer les quelques trois millions de tonnes de matériaux nécessaires à la construction des ouvrages.

Au moment où elle achevait cet axe nord-sud, la Société d'énergie de la Baie James entreprenait la construction d'un axe est-ouest long de $695 \mathrm{~km}$, qui relie entre eux les autres chantiers du complexe. 


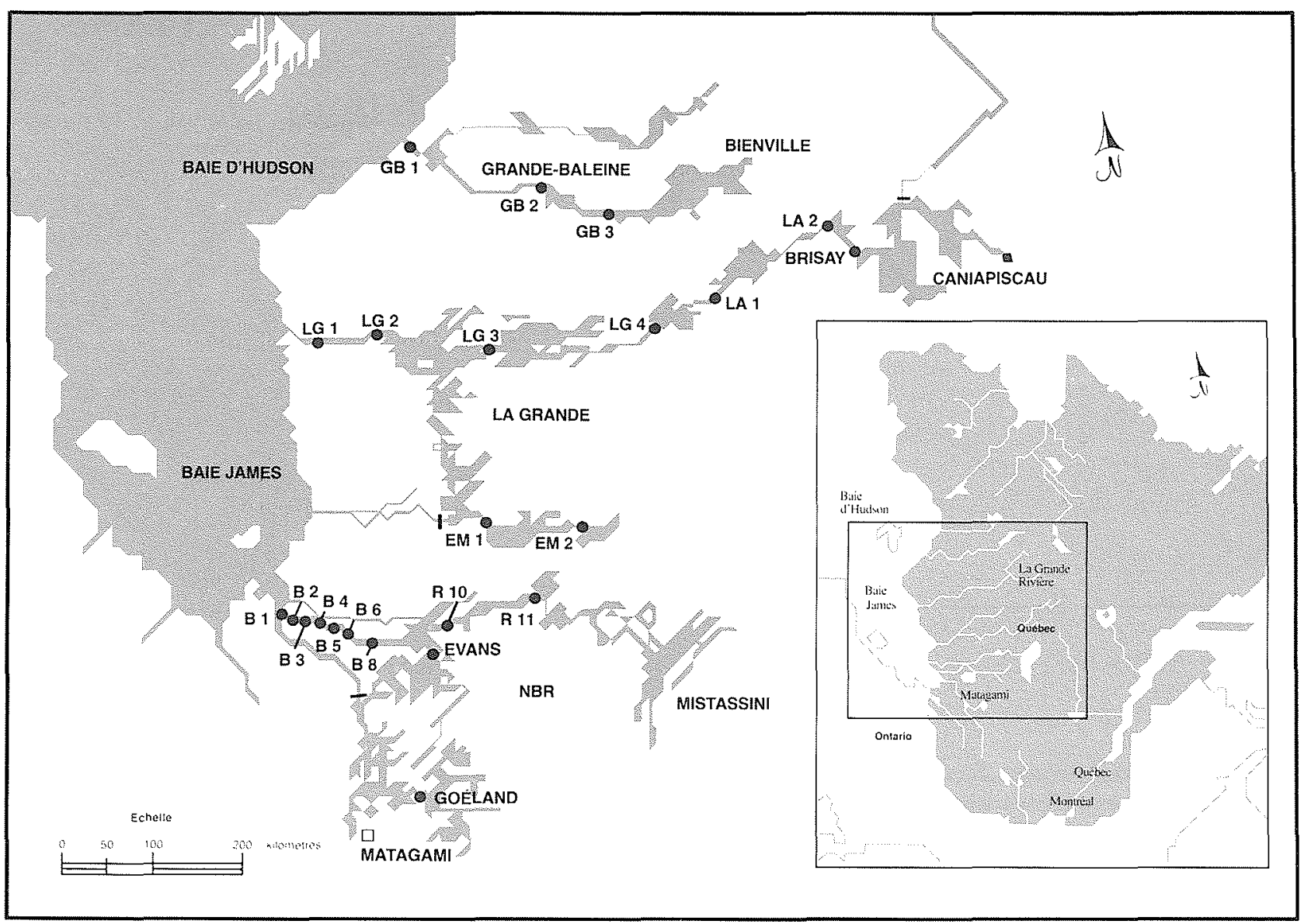

Figure 1

Implantation des différents barrages et centrales constituant l'aménagement hydroélectrique du territoire de la baie James.

\section{Le Complexe La Grande - Phase I}

Le Complexe La Grande - Phase I comprend la construction de trois centrales, La Grande 2, La Grande 3 et La Grande 4, sur La Grande Rivière. Ce cours d'eau, le troisième en importance au Québec, est en réalité un fleuve puisqu'il aboutit à la mer. Il draine un bassin de $98000 \mathrm{~km}^{2}$ et son débit moyen est de $1700 \mathrm{~m}^{3} / \mathrm{s}$. La Grande Rivière coule sur une distance de $800 \mathrm{~km}$ avant d'aller se jeter dans la baie James, et sa dénivelée est de $376 \mathrm{~m}$. En comparaison, le Rhône, qui prend sa source à $1750 \mathrm{~m}$ dans le massif du SaintGothard, parcourt environ la même distance avant d'atteindre la Méditerranée.

Pour accroitre le potentiel de La Grande Rivière, on procède à deux détournements de manière à créer deux réservoirs d'appoint. Ainsi, à environ $150 \mathrm{~km}$ au sud de La Grande Rivière, on détourne $87 \%$ du bassin de l'Eastmain et de ses tributaires, l'Opinaca et la Petite rivière Opinaca, à l'aide d'une série de trois barrages et de huit digues. Le volume d'eau détourné représente un débit régularisé de $840 \mathrm{~m}^{3} / \mathrm{s}$ qui aboutit par gravité au bief d'amont de La Grande 2 par un réseau de lacs et de cours d'eau naturels.

On procède exactement de la même manière avec la rivière Caniapiscau située à l'extrémité nord-est du complexe. A cet endroit, deux barrages et 58 digues sont construits pour dériver $27 \%$ du bassin de la rivière et contenir un réservoir de tête de $4285 \mathrm{~km}^{2}$, qui deviendra le plus grand lac du Québec. Le réservoir Caniapiscau fournira annuellement aux centrales de La Grande Rivière un débit moyen de $780 \mathrm{~m}^{3} / \mathrm{s}$, soit une réserve utile de 38 milliards de $\mathrm{m}^{3}$.

Le Complexe La Grande - Phase I est actuellement réalisé à plus de $85 \%$. En 1985, quand les trois centrales seront terminées, leur puissance sera de $10282 \mathrm{MW}$, et la production annuelle de 62 milliards de $\mathrm{kWh}$ qui, au coût actuel de l'électricité au Québec, rapporteront des revenus bruts de plus de 1,3 milliard de dollars par année (Tableau I).

\section{La Grande 2 (fig. 2)}

Il convient d'abord de signaler que la centrale de La Grande 2, dont la puissance installée s'établit à $5328 \mathrm{MW}$, a été terminée en septembre 1981 avec dix mois d'avance par rapport au calendrier des travaux. A l'heure actuelle, 16 groupes générateurs, comportant chacun une turbine de type Francis accouplée à un alternateur de $333000 \mathrm{~kW}$, fonctionnent à plein rendement.

La salle des machines, immense caverne, haute de $47 \mathrm{~m}$, a été taillée à $140 \mathrm{~m}$ sous la surface du réser- 
voir, dans le massif précambrien dont on a extrait $3225000 \mathrm{~m}^{3}$ de granit.

A La Grande 2, chaque turbine, qui pèse près de 1000 tonnes, est alimentée par une conduite inclinée de $7,9 \mathrm{~m}$ de diamètre et de $180 \mathrm{~m}$ de longueur, à partir d'une prise d'eau située dans une baie du réservoir sur la rive gauche de La Grande Rivière. L'eau, après avoir été turbinée, poursuit sa course vers la chambre d'équilibre, puis est restituée à la rivière par quatre galeries de fuite.

Il est intéressant de noter que les alternateurs sont reliés aux transformateurs installés en surface, par des barres blindées situées dans des puits d'un diamètre de $3,3 \mathrm{~m}$ et d'une hauteur de $123 \mathrm{~m}$. En surface, 24 transformateurs monophasés élèvent la tension de $13,8 \mathrm{kV}$ à $735 \mathrm{kV}$. Ils permettent d'intégrer La Grande 2 au réseau de transport à $735 \mathrm{kV}$ qui achemine vers Montréal l'électricité produite au Complexe La Grande.

Si la centrale de La Grande 2 est remarquable par son gigantisme, il en est de même des autres ouvrages qui ont été construits sur ce chantier. Le barrage a une longueur en crête de $2835 \mathrm{~m}$ et une hauteur de $160 \mathrm{~m}$. Il a nécessité la mise en place de 23 millions de $\mathrm{m}^{3}$ de remblai, soit le même volume que les 30 digues contenant les eaux du réservoir qui s'étend sur $2835 \mathrm{~km}^{2}$.

On a construit à La Grande 2 l'un des plus impressionnants évacuateurs de crues de toute l'histoire du génie civil. Cet ouvrage, qui peut évacuer $15300 \mathrm{~m}^{3} / \mathrm{s}$, est formé d'une partie en béton comportant huit vannes plates de $20,2 \mathrm{~m}$ de hauteur sur 12,2 $\mathrm{m}$ de largeur. A l'aval du coursier, se trouve un canal de $120 \mathrm{~m}$ de largeur qui rejette les eaux vers La Grande Rivière, à $600 \mathrm{~m}$ du barrage. Ce canal, unique en son genre, est excavé dans le roc et formé de 13 paliers d'une hauteur moyenne de $10 \mathrm{~m}$, qui permettent de contrôler et de dissiper l'énergie de l'eau. Tous les produits de déroctage, environ 6,3 millions de $\mathrm{m}^{3}$, ont été utilisés pour la construction du barrage.

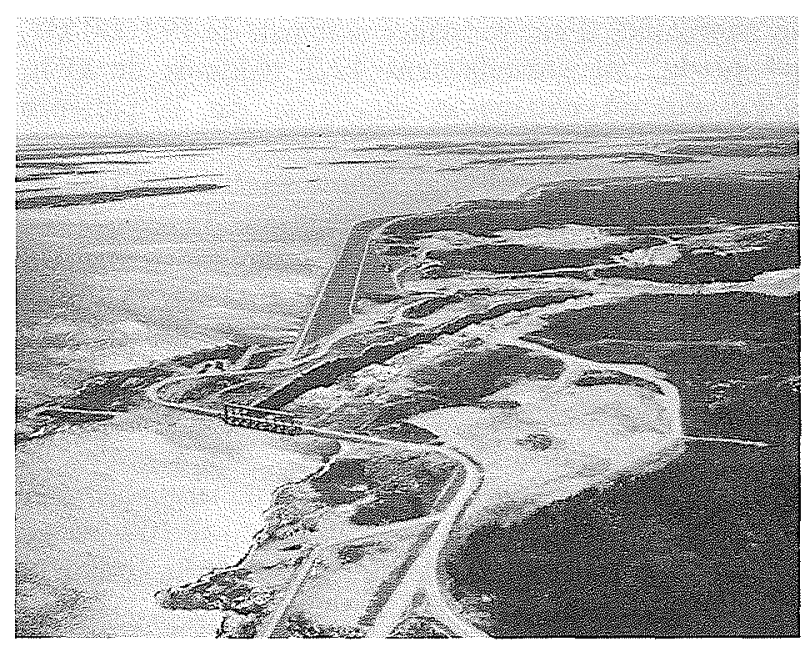

Fig. 2

La Grande 2. - L'aménagement de La Grande 2 impressionne par la masse et la hauteur du barrage, $160 \mathrm{~m}$, et par les 13 marches de l'évacuateur de crues taillées dans le roc et hautes de $10 \mathrm{~m}$. La centrale souterraine peut produire 35,8 milliards de $\mathrm{kWh}$.

\section{La Grande 3 (fig. 3)}

A la Grande 3 aussi, on est en avance sur le calendrier. Le barrage a, en effet, été achevé en octobre 1980, un an plus tôt que prévu. A l'heure actuelle, le réservoir qui fait $2460 \mathrm{~km}^{2}$ est presque plein, et cela, dix mois après sa mise en eau.

\begin{tabular}{|c|c|c|c|c|c|}
\hline \multicolumn{6}{|c|}{ Tableau 1. - Complexe La Grande } \\
\hline Centrale & $\begin{array}{c}\text { Hauteur nominale } \\
\text { de chute } \\
\text { m }\end{array}$ & $\begin{array}{l}\text { Nombre } \\
\text { de groupes } \\
\text { (1) }\end{array}$ & $\begin{array}{c}\text { Puissance } \\
\text { nominale installée } \\
\text { MW }\end{array}$ & $\begin{array}{l}\text { Productibilité } \\
\text { annuelle } \\
\text { moyenne } \\
\text { TWh }\end{array}$ & $\begin{array}{c}\text { Année de mise } \\
\text { en service }\end{array}$ \\
\hline & & \multicolumn{2}{|c|}{ Complexe La Grande-Phase I } & & \\
\hline $\begin{array}{l}\text { La Grande } 2 \\
\text { La Grande } 3 \\
\text { La Grande } 4\end{array}$ & $\begin{array}{r}137,2 \\
79,2 \\
116,7\end{array}$ & $\begin{array}{l}16 \mathrm{~F} \times 333 \\
12 \mathrm{~F} \times 192 \\
9 \mathrm{~F} \times 294,5\end{array}$ & $\begin{array}{l}5328 \\
2304 \\
2650\end{array}$ & $\begin{array}{l}35,8 \\
12,3 \\
14,1\end{array}$ & $\begin{array}{l}1979-1981 \\
1982-1984 \\
1984-1985\end{array}$ \\
\hline La Grande 4 & & $\begin{array}{l}37 \\
\text { Complexe La G }\end{array}$ & $\begin{array}{r}10282 \\
\text { de - Phase II }\end{array}$ & 62,2 & \\
\hline $\begin{array}{l}\text { La Grande } 1 \\
\text { Brisay } \\
\text { Laforge } 1 \\
\text { Laforge } 2 \\
\text { Eastmain }\end{array}$ & $\begin{array}{l}28,2 \\
38,4 \\
58,4 \\
26,0 \\
65,0\end{array}$ & $\begin{array}{r}10 \mathrm{H} \times 114,0 \\
2 \mathrm{~K} \times 214,0 \\
6 \mathrm{~F} \times 136,6 \\
3 \mathrm{H} \times 106,0 \\
3 \mathrm{~F} \times 183,3\end{array}$ & $\begin{array}{r}1140 \\
428 \\
820 \\
318 \\
525\end{array}$ & $\begin{array}{l}7,0 \\
2,3 \\
4,2 \\
1,7 \\
2,9\end{array}$ & $\begin{array}{c}1989.1990 \\
1989 \\
1990 \\
1992 \\
1993\end{array}$ \\
\hline & & 24 & 3231 & 18,1 & \\
\hline
\end{tabular}

(1) $F=$ Francis $\quad H=$ Hélice $K=$ Kaplan 
Etablie à $117 \mathrm{~km}$ à l'amont de La Grande 2, la centrale de La Grande 3 est nichée dans un tranchée profonde excavée au pied du barrage. Elle est équipée de douze turbines de type Francis entraînant chacune un alternateur d'une puissance nominale de $192000 \mathrm{~kW}$, qui produiront annuellement 12,3 milliards de $\mathrm{kWh}$.

Le site du barrage se caractérise par la présence d'une île au milieu de La Grande Rivière qui se trouve ainsi partagée en deux bras. D'une hauteur de $100 \mathrm{~m}$, le barrage de La Grande 3 est le plus long du complexe : sa longueur en crête est de $3,8 \mathrm{~km}$. Sa construction a nécessité un volume total de remblai de 22,2 millions de $\mathrm{m}^{3}$. Il a aussi fallu mettre en place 12 millions de $\mathrm{m}^{3}$ de matériaux naturels pour réaliser les 67 digues qui circonscrivent le réservoir.

L'évacuateur de crues comporte un gigantesque saut de ski en béton. Cet ouvrage remarquablement esthétique a été conçu pour évacuer $10100 \mathrm{~m}^{3} / \mathrm{s}$.

Le premier groupe turbine-alternateur de La Grande 3 a commencé à produire le 20 juin dernier et les onze autres groupes seront mis en service à des intervalles de moins de deux mois.

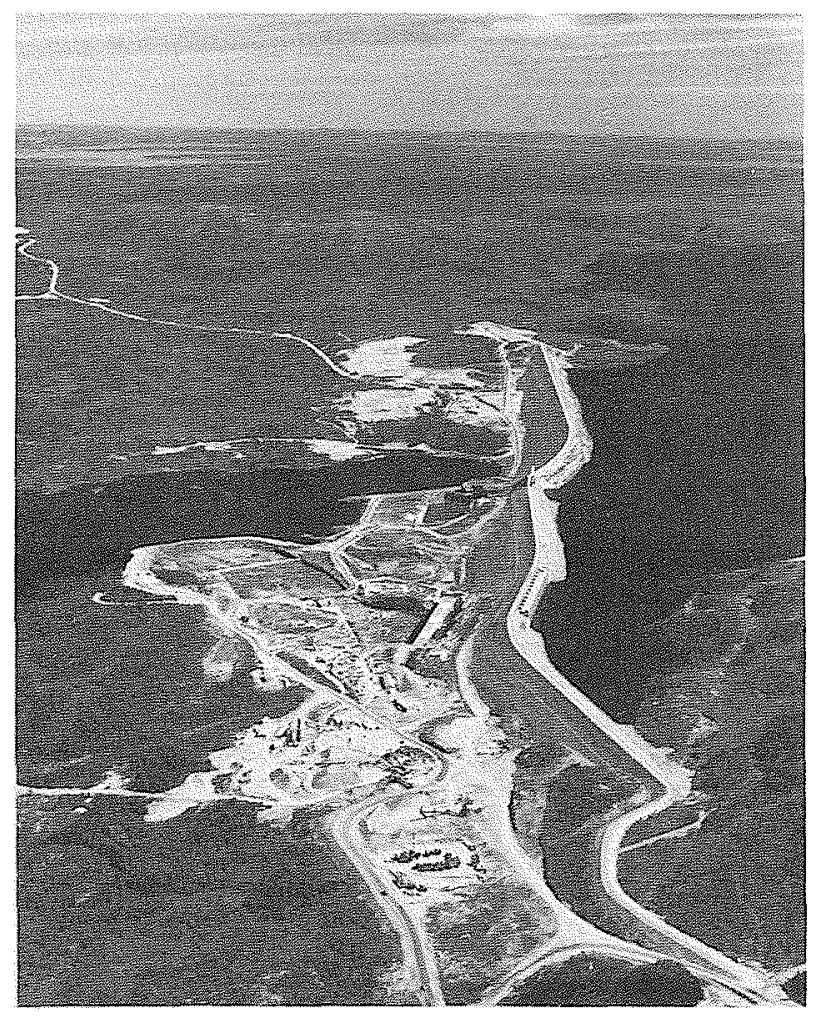

Fig. 3

La Grande 3. - Le barrage le plus long du Québec - tout près de $4 \mathrm{~km}$ - coupe La Grande Rivière à la hauteur de La Grande 3. Au centre, excavé sur la pointe d'un ñe rocheuse maintenant submergée, l'évacuateur de crues. Au pied du barrage, en rive droite, la centrale d'une puissance de 2304 MW.

\section{La Grande 4 (fig. 4)}

A $125 \mathrm{~km}$ en amont de La Grande 3, on construit la troisième et dernière centrale comprise dans la première phase du complexe : La Grande 4.
Sur ce chantier, on a franchi une autre étape marquante en septembre 1981. Les travailleurs ont en effet célébré la fin de la construction du barrage avec une avance de quinze mois.

Long de $3600 \mathrm{~m}$, haut de 125 , cet ouvrage a nécessité la mise en place de 20 millions de $\mathrm{m}^{3}$ de remblai. Quant aux huit digues qui représentent un volume additionnel de 14 millions de $\mathrm{m}^{3}$ de matériaux, elles sont pratiquement terminées.

La centrale de La Grande 4 est, elle aussi, construite en surface. Avec une puissance installée de $2637 \mathrm{MW}$, elle sera en importance la deuxième centrale du Québec, après "La Grande 2". Sa mise en service est prévue pour février 1984 , mais elle n'atteindra son plein rendement qu'en 1985, produisant annuellement 14,1 milliards de $\mathrm{kWh}$.

\section{Le détournement Eastmain}

Le chantier où l'on effectuait des travaux de détournement de l'Eastmain, de l'Opinaca et de la Petite rivière Opinaca, est fermé depuis octobre 1980. Désormais, à chaque seconde, $840 \mathrm{~m}^{3}$ d'eau viennent accroître le potentiel hydraulique de La Grande 2 .

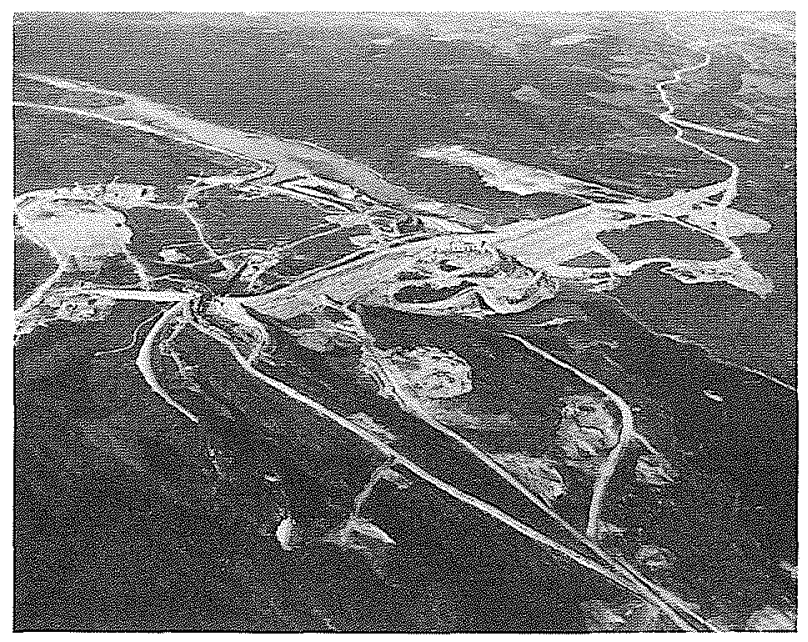

Fig. 4

La Grande 4. - Un barrage, allongé pour bloquer une vallée secondaire, dompte La Grande Rivière à la hauteur de La Grande 4. Au centre, la prise d'eau d'où partent les conduites forcées qui débouchent dans la centrale en aval. A l'extrémité sud, l'évacuateur de crues qui traverse un lac naturel avant de rejoindre La Grande Rivière.

En moins de trois ans, huit digues et trois barrages $\mathrm{y}$ ont été construits et à l'un d'eux, la profondeur du mort-terrain aura permis d'expérimenter avec succès des techniques de pointe lors du traitement des fondations : la tranchée de boue et la paroi de béton moulée.

\section{Le détournement Caniapiscau (fig. 5)}

$\mathrm{Au}$ dernier chantier, celui de Caniapiscau, la mise en eau est chose faite depuis octobre 1981. Le remplissage du réservoir durera deux ans et le plan d'eau ainsi créé aura une superfice de $4285 \mathrm{~km}^{2}$. 
Sur ce chantier, qui s'étale sur une distance de $180 \mathrm{~km}$, les travaux auront duré sept ans. Durant la période de pointe (1980-1981), il aura fallu recourir à plus de 3300 travailleurs répartis dans cinq campements.

Ce tour d'horizon aura sans doute permis de percevoir l'ampleur des travaux exécutés au Complexe La Grande - Phase I.

Il faut ici souligner que dès le départ, le maître d'œuvre a été conscient des répercussions écologiques et sociologiques qu'entraîneraient les travaux.

A cet effet, la Société d'énergie de la Baie James a énoncé une politique claire de protection de l'environnement et adopté des règlements et des directives internes qui lui permettent d'intervenir à chacune des étapes de la réalisation des ouvrages. Elle a ainsi entrepris divers travaux de correction, qui vont de la construction de passes migratoires au relogement des populations autochtones en passant par l'ensemencement aérien des berges des rivières exondées et le reboisement des sites touchés par les travaux. A ce jour, près de 50 millions de dollars ont été consacrés à la protection et au réaménagement de l'environnement, ce qui fait du Complexe La Grande un des rares projets cités favorablement par les organismes à vocation écologique.

\section{Le Complexe La Grande - Phase II}

L'aménagement de la seconde phase du complexe La Grande consiste à exploiter la dénivellation non négligeable que possèdent le cours supérieur de la rivière Eastmain, le parcours des eaux entre le réservoir Caniapiscau et le bief d'amont de La Grande 4, et enfin le cours inférieur de La Grande Rivière.

Cinq sites de centrales ont été jugés économiquement rentables. La centrale d'Eastmain 1 sera implantée sur le cours supérieur de la rivière Eastmain, alors que trois autres centrales, Brisay, Laforge 2 et Laforge 1, seront construites sur le parcours des eaux détournées depuis le réservoir Caniapiscau jusqu'à La Grande 4.

La centrale de La Grande 1, à $37 \mathrm{~km}$ de l'embouchure de La Grande Rivière, complète enfin cette phase qui ajoutera plus de $3200 \mathrm{MW}$ à la puissance installée d'Hydro-Québec (Tableau I). Il va sans dire que sa construction nécessitera la mise en place de toute une infrastructure : $300 \mathrm{~km}$ de routes, cinq campements, deux aéroports et trois villages familiaux.

\section{Le Complexe Grande-Baleine}

Le Complexe Grande-Baleine sera implanté sur la Grande rivière de la Baleine. Celle-ci chevauche le $55^{\mathrm{e}}$ parallèle ce qui la situe à peu près à la même latitude que Moscou. Avec son bassin versant de $43500 \mathrm{~km}^{2}$, c'est le plus important cours d'eau à se jeter dans la baie d'Hudson. Depuis sa source jusqu'à la baie, la rivière parcourt une distance de $370 \mathrm{~km}$ avec une dénivelée de $400 \mathrm{~m}$.

Le schéma d'aménagement prévoit la construction de trois centrales sur la Grande rivière de la Baleine

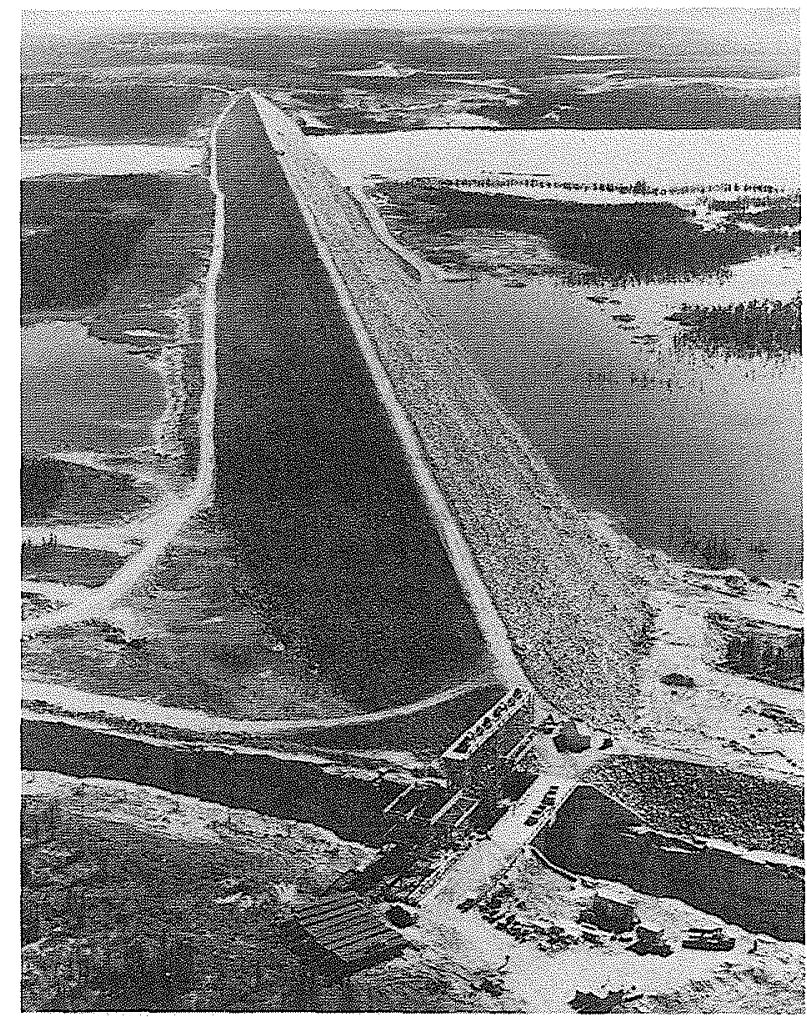

Fig. 5

Caniapiscau. - Le barrage de KA 3, d'une longueur de $3 \mathrm{~km}$, coupe le bras est de la rivière Caniapiscau. Il est terminé depuis 1980. A son extrémité ouest, l'évacuateur de crues qui sert de soupape de sûreté au réservoir de Caniapiscau. Il peut laisser s'échapper jusqu'à $3700 \mathrm{~m}^{3}$ d'eau par seconde.

vers laquelle seront dérivées, au niveau du futur emplacement de la centrale de Grande-Baleine 1, les eaux de la Petite rivière de la Baleine qui coule un peu plus au nord, parallèlement à la première. On mettra ainsi à contribution près de $80 \%$ du débit naturel des deux cours d'eau.

Trois retenues seront créées, dont la plus importante est celle du lac Bienville. Trois évacuateurs de crues et un ouvrage régulateur, à la sortie du lac Bienville, compléteront cet aménagement dont la puissance installée s'élèvera à $2900 \mathrm{MW}$, grâce aux 11 groupes turbinesalternateurs répartis dans les trois centrales : GrandeBaleine 1, Grande-Baleine 2 et Grande-Baleine 3 (Tableau II).

A la pointe des travaux, l'effectif totalisera plus de 6000 travailleurs qui devront unir leurs efforts pendant neuf ans, soit de 1983 à 1991 inclusivement. Pour les accueillir, il faudra prévoir toute une infrastructure qui comprendra notamment, trois campements, deux villages familiaux et des campements satellites.

Selon les études faites à ce jour, les conditions géologiques sont très favorables et les matériaux d'emprunt sont en général disponibles à proximité des chantiers.

Située dans une vallée à quelques kilomètres de la baie d'Hudson, la centrale de Grande-Baleine 1 sera équipée de cinq groupes Francis d'une puissance totale installée de $1978 \mathrm{MW}$. Elle sera construite sous terre et la chute brute maximale sera de $193 \mathrm{~m}$. La retenue 


\begin{tabular}{|c|c|c|c|c|c|}
\hline \multicolumn{6}{|c|}{ Tableau II } \\
\hline \multirow[t]{2}{*}{ Centrale } & $\begin{array}{l}\text { Hauteur nominale } \\
\text { de chute } \\
\text { m }\end{array}$ & $\begin{array}{c}\text { Nombre } \\
\text { de groupes } \\
\text { (1) }\end{array}$ & $\begin{array}{c}\text { Puissance } \\
\text { nominale installée } \\
\text { MW }\end{array}$ & $\begin{array}{l}\text { Productibilité } \\
\text { annuelle } \\
\text { moyenne } \\
\text { TWh }\end{array}$ & $\begin{array}{c}\text { Année de mise } \\
\text { en service }\end{array}$ \\
\hline & & \multicolumn{2}{|c|}{ Complexe Grande-Baleine } & & \\
\hline Grande-Baleine 1 & 191,5 & $5 F \times 395,6$ & 1978 & 10,3 & 1990 \\
\hline Grande-Baleine 2 & 90,7 & $3 F \times 153,3$ & 460 & 2,4 & 1991 \\
\hline \multirow[t]{3}{*}{ Grande-Baleine 3} & 97,0 & $3 \mathrm{~F} \times 151,0$ & 453 & 2,4 & 1991 \\
\hline & & 11 & 2891 & 15,1 & \\
\hline & \multicolumn{4}{|c|}{ Complexe Nottaway-Broadlback-Rupert (NBR) } & \\
\hline Goéland & 18,4 & $3 \mathrm{~K} \times 66,7$ & 200 & 0,94 & 1995 \\
\hline Evans & 14,9 & $5 \mathrm{~K} \times 55,0$ & 275 & 1,46 & $1993-1994$ \\
\hline Rupert 11 & 33,6 & $2 \mathrm{~K} \times 150,0$ & 300 & 1,55 & 1992 \\
\hline Rupert 10 & 36,4 & $3 \mathrm{~K} \times 123,3$ & 370 & 2,11 & 1992 \\
\hline Broadback 8 & 27,7 & $6 \mathrm{H} \times 116,7$ & 700 & 4,65 & 1993-1995 \\
\hline Broadback 6 & 36,6 & $6 \mathrm{H} \times 155,0$ & 930 & 6,20 & 1993-1995 \\
\hline Broadback 5 & 68,2 & $10 \mathrm{~F} \times 185,5$ & 1855 & 11,86 & $1994-1996$ \\
\hline Broadback 4 & 21,1 & $6 \dot{H} \times 82,5$ & 495 & 3,57 & 1994-1996 \\
\hline Broadback 3 & 31,1 & $6 \mathrm{H} \times 133,3$ & 800 & 5,32 & $1996-1997$ \\
\hline Broadback 2 & 30,3 & $6 \mathrm{H} \times 128,3$ & 770 & 5,13 & $1997-1998$ \\
\hline \multirow[t]{2}{*}{ Broadback 1} & 23,0 & $6 \mathrm{H} \times 93,3$ & 560 & 3,69 & \\
\hline & & 59 & 7255 & $\overline{46,46}$ & \\
\hline
\end{tabular}

(1) $F=$ Francis $H=$ Hélice $K=$ Kaplan

du bief d'amont sera obtenue grâce à la construction de 29 digues et de deux barrages.

Pour construire la centrale, l'évacuateur de crues, d'une capacité de $4050 \mathrm{~m}^{3} / \mathrm{s}$, et les autres ouvrages de Grande-Baleine 1, il faudra mettre en place $165000 \mathrm{~m}^{3}$ de béton. Les travaux devraient débuter vers la fin de 1984.

La centrale de Grande-Baleine 2 sera également construite sous terre, à $225 \mathrm{~km}$ de l'embouchure de la rivière. Elle sera dotée de trois groupes d'une puissance totale de $460 \mathrm{MW}$ sous une chute brute de $92 \mathrm{~m}$. Les eaux du bief d'amont seront retenues grâce à un barrage et à un réseau de dix digues Le début des travaux est prévu pour le printemps 1985 .

Au total, on excavera 2,5 millions de $\mathrm{m}^{3}$ de mortterrain et de roc en surface et sous terre. Quant au remblai, destiné à la construction du barrage et des digues, son volume atteindra 3,3 millions de $\mathrm{m}^{3}$. Enfin, $75000 \mathrm{~m}^{3}$ de béton seront utilisés pour construire la centrale et l'évacuateur de crues.

A la fin de 1986, au km 290, devrait commencer la construction de la centrale de Grande-Baleine 3 qui comptera trois groupes d'une puissance totale installée de $453 \mathrm{MW}$.

$\mathrm{Au}$ total, 23 digues et deux barrages contiendront les eaux du réservoir. Les excavations de mort-terrain, de roc en surface et sous terre totaliseront 4,4 millions de $\mathrm{m}^{3}$. Les digues et les barrages représenteront 5,6 millions de $\mathrm{m}^{3}$ de remblai et on coulera $68000 \mathrm{~m}^{3}$ de béton.

A ces trois centrales viendront s'ajouter le réservoir Bienville et le détournement de la Petite rivière de la Baleine. Afin de rehausser le lac Bienville de près de $8 \mathrm{~m}$, il faudra construire un ouvrage de contrôle d'une capacité de $1310 \mathrm{~m}^{3} / \mathrm{s}$ et disposer dans les vallées 45 digues d'une longueur totale, en crête, de $16,5 \mathrm{~km}$. La hauteur de ces digues variera de un à neuf mètres. Les travaux devraient débuter en 1987.

Pour canaliser l'eau vers la centrale de GrandeBaleine 1 , on détournera la Petite rivière de la Baleine au moyen d'un réseau de 22 digues, d'une longeur totale de près de $6 \mathrm{~km}$, et dont la hauteur variera de 2 à $18 \mathrm{~m}$. Comparativement à d'autres sites, le mortterrain excavé représente un faible volume : $130000 \mathrm{~m}^{3}$ seulement. Le remblai, d'autre part, atteindra les $860000 \mathrm{~m}^{3}$

On aura accès à ces ouvrages par une route permanente dont la longueur est estimée à plus de $500 \mathrm{~km}$. Le transport aérien permettra également d'acheminer vers les chantiers la main-d'œuvre, une partie du matériel et des matériaux. Deux aéroports sont prévus, l'un dans la région de Grande-Baleine 1 et l'autre entre GrandeBaleine 2 et Grande-Baleine 3. Des pistes de glace et des pistes temporaires seront également aménagées à l'emplacement des digues éloignées, soit aux lacs Saindon, Mollet et Elizabeth. 
Selon le programme d'équipement soumis par HydroQuébec au Gouvernement en novembre dernier ${ }^{(1)}$, la mise en service des premières turbines aurait lieu dès 1990. Par conséquent, la construction de la route vers Grande-Baleine 1 commencera dès l'automne 1982 et le gros des travaux à l'été 1985. C'est dire l'énormité de la tâche à accomplir et le long chemin qu'il reste encore à parcourir avant la mise en service de ces équipements.

\section{Le Complexe Nottaway-Broadback-Rupert (NBR)}

A environ $300 \mathrm{~km}$ au sud de La Grande Rivière qui, rappelons-le, comprend l'aménagement des phases I et II du Complexe La Grande, se trouvent les bassins versants de trois rivières : la Nottaway, la Broadback et la Rupert. D'où le nom du Complexe NBR.

L'aménagement de ces bassins s'avère intéressant car les trois cours d'eau considérés convergent vers la baie James, et leur dénivellation est concentrée en aval, soit dans les derniers $80 \mathrm{~km}$ de leur parcours.

En combinant les apports des trois rivières par des canaux relativement courts et en installant la plus grande partie des équipements sur le tronçon inférieur de la Broadback, on pourra produire annuellement près de 47 milliards de $\mathrm{kWh}$, soit environ les deux tiers de la production du Complexe La Grande $\bar{P}$ hase $\overline{\mathrm{I}}$. Une telle production représente un équivalent thermique de 43 millions de barils de pétrole ; évaluée à $32 \$$ US le baril, elle équivaut à 1,4 milliard de dollars US (1981) et constitue une somme que le Québec n'aura pas à dépenser à l'extérieur de ses frontières pour se procurer une énergie non renouvelable.

Onze centrales hydroélectriques seront construites dont sept sur le cours inférieur de la rivière Broadback.
Quatre autres centrales, de plus faible puissance, seront situées à la sortie des réservoirs périphériques. La puissance installée totalisera $7255 \mathrm{MW}$ (Tableau II).

La régularisation des eaux des trois bassins versants nécessitera la création de cinq grandes retenues. Ce n'est pas moins de 19 barrages et 118 digues qu'il faudra ériger pour retenir les eaux de ces trois rivières.

Les travaux du Complexe NBR s'échelonneront entre 1986 et 1999 . A la pointe des travaux, environ 20000 personnes devront être logées et nourries sur les divers chantiers.

La route Matagami - La Grande 2 traverse le territoire du Complexe NBR par le centre. C'est sur cet axe que viendront se greffer les embranchements qui desserviront les chantiers des centrales et des réservoirs situés dans la partie sud du complexe. Il faudra mettre en place un réseau routier comprenant $410 \mathrm{~km}$ de routes d'accès et $225 \mathrm{~km}$ de routes d'hiver. Les travaux débuteront en 1985.

\section{Conclusion}

Le tableau qui vient d'être brossé des projets d'aménagement hydroélectrique du territoire de la Baie James aura sans doute permis de saisir l'ampleur des travaux en cours et de rendre compte des efforts consentis par la collectivité québécoise pour s'assurer une source d'énergie propre et renouvelable.

D'un territoire hostile, les pionniers de la Baie James, hommes et femmes du Québec, auront bientôt réussi à tirer $10000 \mathrm{MW}$ qui seront versés au réseau d'HydroQuébec. L'avenir immédiat consiste à aménager des rivières dont les bassins versants couvrent une superficie presque équivalente à celle de la Phase I du Complexe La Grande, et la Société d'énergie de la Baie James s'apprête à en relever les prochains défis.
(1) Depuis la présentation de cette communication, une révision des besoins énergétiques du Québec a conduit à reporter de quelques années tous les ouvrages qui ne sont pas présentement en cours de construction. 


\section{Discussion}

Président : M. LONDE

M. le Président. - Merci, Monsieur MARINIER. Pour ma part, j'ai un peu le vertige devant l'ampleur du programme et aussi devant la vitesse de réalisation.

Ce sera peut-être ma question pour lancer les débats.

Vous nous avez dit à deux reprises que les aménagements avaient été achevés de nombreux mois avant leur date contractuelle. Quel en est le mécanisme et quelle en est la raison?

M. MARINIER. - D'abord, on pourrait être tenté de dire que le programme était mal fait!

En fait, le programme a été établi au cours de l'année 1975 et il correspondait au rythme alors atteint pour les grands travaux de la Baie James; en effet, on avait estimé des délais et les entrepreneurs étant nouveaux dans le territoire, ils ne connaissaient pas tout à fait le rythme auquel ils pouvaient travailler. L'expérience nous a conduit à réviser les tracés, les échéanciers et à réaliser de bonnes économies, parce que chez nous, les chantiers étant saisonniers, nous évitions plusieurs mois de frais fixes si nous réussissions à ne pas revenir l'année suivante.

Ce sont les incertitudes du début et les bonnes performances des entreprises qui ont permis de raccourcir les temps de réalisation.

M. le Président. - Comme vous avez fait votre exposé plus rapidement que prévu au programme, nous pouvons ouvrir la discussion avant la projection du premier film.

Qui demande la parole?

M. HOEPPENER. - Est-il possible de travailler toute l'année sur les chantiers de la Grande, en raison des conditions climatiques et géologiques?

M. MARINIER. - Je dois d'abord mentionner que la plupart des travaux sont d'énormes remblais - comme vous avez pu le constater - dont l'étanchéité est réalisée avec de la moraine glaciaire, ce matériau, sable silteux un peu spécial, que Dieu a mis à notre disposition dans le Nord, sinon, il n'aurait pas été possible de réaliser ces remblais. Cette moraine est très près de son point de saturation et ne peut être manipulée ou mise en place que durant 90 jours par an en période estivale, ce qui limite la réalisation du noyau. Pour les gros enrochements, il $n^{\prime} y$ a pas de problème, mais ils doivent suivre le rythme de mise en place de la partie centrale des barrages.

Pour les travaux souterrains, il n'y a pas de difficultés : ils peuvent être exécutés toute l'année à l'exception des travaux à ciel ouvert.

Il y a toutefois la période des fêtes - de mi-décembre à mijanvier - où les travaux tournent au ralenti; il n'y a alors que quelques personnes sur place pour assurer l'entretien, donc un minimum de personnel. En gros, un chantier de 800 personnes en hiver en compte 4000 en été, et ceci pendant plusieurs années de suite.

M. Le Président. - Un masque amont en béton aurait peut être résolu le problème mais, sans doute, y a-t-il aussi un problème de disponibilité de mois dans l'année.

M. MARINIER. - Cette question a été examinée dès le début du projet vu qu'elle peut s'appliquer à de grands ouvrages (pour La Grande 2, par exemple où la hauteur est de $160 \mathrm{~m}$ ). Les projeteurs ont fait le tour du monde et de la question pour finalement, ne pas s'engager dans cette voie pour le premier ouvrage. Cette approche a donné le ton pour les autres barrages et nous a familiarisés avec une construction en moraine qui est plus souple et qui cause moins de surprises.

M. le Président. - Y a-t-il encore une question avant de passer le film?

M. COTILLON. - J'ai deux questions très courtes à poser.

Voici la première : quand vous parlez de dollars, s'agit-il de dollars canadiens ou de dollars américains?
M. MARINIER. - Ce sont des "petits" dollars, c'est-à-dire des dollars canadiens qui valent présentement $5 / 6$ du dollar américain.

M. COTILLON. - Deuxième question : pourquoi n'avezvous pas commencé par le Sud, c'est-à-dire par N.B.R., et êtesvous passés tout de suite à La Grande?

M. MARINIER. - C'est une très bonne question. C'était tellement plus logique, en effet, d'agir ainsi dès le début. Malheureusement, le complexe N.B.R., qui est très facile dans sa partie supérieure, devient compliqué dans la partie basse à cause de la présence d'argiles sensibles. Aujourd'hui, après plusieurs années d'essais et de prospections, on sait que ce sera un terrain très difficile qui obligera à des techniques de construction qu'on n'a pas encore tout à fait maîtrisées et à des traitements de fondations très coûteux. Devant cette situation, les autorités ont préféré commencer par la rivière La Grande, qui ressemble beaucoup aux aménagements déjà réalisés; nature de rocher, moraines, etc... C'était plus sûr et cela s'est avéré beaucoup plus facile que prévu.

M. le Président. - Si vous êtes d'accord, M. MARINIER, nous pouvons maintenant faire projeter le premier film.

M. MARINIER. - Le film s'intitule "Terre d'énergie"; c'est un résumé du complexe qui dure 18 minutes. Ensuite, on fera une pause et une discussion suivra si des questions sont posées, puis on projettera le second film, d'une durée de 18 minutes également et qui est un film d'entreprise fait avec du matériel d'archives. Ce film intitulé "L'eau montre à L G 3 " est néanmoins très intéressant et montre l'évolution du site depuis le tout début du chantier en 1974 jusqu'à Septembre 1981.

(Projection du premier film)

M. Le Président. - Le mot de la fin du fïm résume bien la situation. Les principes n'ont pas changé, mais l'échelle en elle-même est votre grand problème.

Nous continuons la discussion avant de présenter le dernier film, ce qui sera fait dans vingt minutes environ.

J'ai, pour ma part, une question à poser. Monsieur MARINIER, vous avez devant vous un grand nombre d'ouvrages à réaliser très vite. Avez-vous essayé de standardiser certains de ces aménagements?

M. MARINIER. - Présentement, la première partie du complexe a été normalisée dans le domaine des grandes vannes planes. Vous avez peut-être remarqué qu'il n'y a pas de vannes secteurs, mais plutôt des vannes planes qu'on a modulées à environ $15 \mathrm{~m}$ de largeur par vanne. Nous avons fait un effort pour normaliser également les vannes batardeaux, les jeux de poutrelles des prises d'eau et les diffuseurs des centrales.

Présentement, avec le petit recul dont on dispose, on envisage une normalisation dans les centrales, surtout en vue d'aménagements qui porteront sur 11 centrales de moyenne ou basse chute et tout particulièrement sur une certaine normalisation des turbines, voire même des déchargeurs en remplacement des vannes d'évacuateur, lesquelles sont déjà toutes semblables.

Cet effort de normalisation sera plus poussé encore pour le complexe N.B.R. à la lumière de ce qui aura pu être réalisé dans le complexe La Grande - phase 1.

M. AMARANTE. - Au Québec, vous avez une situation presque semblable à celle du Brésil : pour les aménagements hydroélectriques, les critères d'utilisation sont probablement les mêmes qu'au Brésil.

Je voudrais savoir au sujet du dimensionnement du complexe La Grande, si vous avez considéré la production de pointe ou si au contraire les ouvrages ont été conçus pour satisfaire les besoins de base en énergie et la production de 
pointe étant assurée par des installations spéciales au voisinage des grands centres de charge que sont QUEBEC et MONTREAL.

C'est un problème économique et je voudrais savoir comment il a été résolu.

M. MARINIER. - Je dois dire que cela s'est fait en deux temps. La première réaction a été celle de tout hydraulicien qui ne conçoit pas qu'un tel potentiel, situé à $1000 \mathrm{~km}$ du point de consommation, ne puisse être aménagé autrement qu'en usine de base; et on a adopté un facteur d'utilisation d'environ $80 \%$. Les premiers projets ont été conçus de cette façon. Mais on s'est vite aperçu que pour disposer rapidement d'une source de puissance, il valait mieux baisser le facteur d'utilisation. La centrale LG 2 étant suffisamment avancée n'a pas pu être modifiée, mais par contre l'équipement de LG 3 est passée de 10 à 12 groupes pour faire baisser son facteur d'utilisation à environ $61 \%$. Celui de la centrale LG 4 est passé de 7 à 9 groupes et il est prévu qu'il y aura une production de pointe dans la majorité des autres centrales programmées dans l'ensemble du complexe.

Ajoutons qu'au QUEBEC, il y a très peu d'usines de pointe il $\mathrm{y}$ a quelques groupes thermiques, à gaz, dans la banlieue de MONTREAL, et on construit présentement une centrale souterraine d'une puissance additionnelle de $1000 \mathrm{MW}$ à Manic 5.

M. COTILLON. - Au point de nue exploitation, quel est le système que vous avez envisagé ? Est-ce une télécommande ou des commandes par le personnel permanent sur place? Qu'en est-il du personnel d'usine et de surveillance des lignes?

M. MARINIER. - La télécommande est prévue pour l'ensemble du réseau du complexe de La Grande et comme nous en sommes présentement à la phase des essais et des mises en route, il y a beaucoup de monde. Pour le moment, la télécommande ne fonctionne pas tout à fait, mais les équipements, les ordinateurs sont là; le tout rassemblé dans une salle de contrôle située à LG 2 d'où l'on pourra mettre en route n'importe quel groupe du complexe.

Pour le personnel qui devra assurer l'exploitation de ces centrales, HYDRO-QUEBEC avait envisagé jusqu'à présent la formule $8-6$, c'est-à-dire 8 jours de travail et 6 jours de congé, aller-retour en avion. Cela semblait être le paradis comparé aux formules avec résidence sur place qui sont devenues très coûteuses. Actuellement, on pense à une autre formule : 20 jours de travail, 20 jours de repos. C'est un domaine très complexe qui touche aux relations avec les syndicats, domaine dans lequel l'ingénieur ne peut intervenir et pour lequel on a recours à des spécialistes. Présentement, on applique la formule 8-6.

M. COTILLON. - Au total, combien y a-t-il de personnel?

M. MARINIER. - Acutellement, on déplace de 100 à 110 personnes mais ce n'est pas encore la pleine exploitation. Dans ce nombre sont incluses les personnes qui sont occupées pour les travaux mêmes et celles qui assurent la surveillance et l'inspection des barrages. Dans quelques années, ce personnel de surveillance sera réduit tandis que le personnel d'exploitation s'accroîtra.

M. CLERDOUET. - Avant d'exploiter, pour projeter et construire les projets annoncés, avez-vous évaluer le risque de manque d'hommes, en techniciens, et en ouvriers qualifiés?

M. MARINIER. - Tout dépend du calendrier. En 1979, il a fallu sur place quelque 18000 personnes, ce qui a été assez difficile à recruter puisqu'un certain nombre de travailleurs ont été recrutés hors du Québec. Pour les bureaux d'études, il n'y a pas eu de problème, car il y a à MONTREAL une concentration de bureaux spécialisés en aménagements hydroélectriques; ce qui est un peu unique au monde. De tels bureaux comme Montreal Engineering, le Groupe SNC, le Groupe Lavalin, etc... dont l'ensemble comprend de 3000 à 4000 ingénieurs sont orientés surtout vers les grands traveaux de génie civiel. Présentement, cet effectif est en baisse et nous sommes au "creux de la vague" parce que l'accroissement de la demande en électricité qui était de $7 \%$ l'an au Québec, est tombée, à 3 ou $4 \%$, de sorte que les travaux annoncés et qui devaient commencer cette année, ne démarreront qu'en 1983.
On examine aussi la possibilité d'exporter des blocs d'énergie en Nouvelle Angleterre. Je lisais dans les journaux d'hier que M. LEVESQUE, notre Premier Ministre, se rendra prochainement à NEW YORK pour négocier cette affaire qui peut représenter pour SEBJ un ou deux ans de travail.

M. CLERDOUET, - et pour les chantiers?

M. MARNIER. - Il n'y a plus de problème : le personnel est sur place. Il y a eu des remous au début, mais maintenant, les difficultés sont aplanies : $36 \%$ des travailleurs en sont à leur deuxième ou troisième campagne annuelle. Il faut dire qu'on a dépensé beaucoup dans le confort et les loisirs : des cinémas, des piscines olympiques, des jeux de bowling et de curling, etc. tout y est gratuit ou presque pour les employés, y compris le gite et le couvert qui sont fournis par la Sociéte d'Energie.

M. AMARANTE. - Nous avons vu dans le film des photographies sous la neige. Quelle température fait-il l'hiver et combien de temps dure cette froidure?

M. MARINIER. - Dans le cycle des saisons, la neige au sol disparaît à la mi-mai et réapparait autour du ler Septembre, ce qui toutefois n'empêche pas la poursuite de certains travaux pour la mise en place de matériaux argileux comme la moraine, il faut choisir à partir du mois de Septembre, le bon aprèsmidi. Dans le film, tourné en Octobre, la neige au sol est permanente. Les minima de température au moment des Fêtes, début janvier, se situent aux environs de $-30^{\circ} \mathrm{C}$, avec des nuits claires à -40 ou $-50^{\circ} \mathrm{C}$. C'est pour cette raison que tout est ralentit en hiver ; les travaux extérieurs cessent et les travaux souterrains sont réduits au minimum tout en maintenant le camp en opération afin de permettre une meilleure reprise en mars et avril.

M. HAMON. - Je reviens en arrière, à propos des vannes. M. MARINIER pourrait-il inđiquer les raisons qui, pour l'équipement des évacuateurs de crues, ont fait préférer les vannes planes aux vannes secteurs.

M. MARINIER. - C'est en raison de la mauvaise expérience des vannes secteurs. Peu de vannes-secteurs ont été construites au Québec et une installation sur le fleuve Saint-Laurent, en particulier, nous a crée des problèmes. D'abord, ayant très peu de constructeurs de ce type de vannes, il a été difficile d'obtenir un bon produit qui, de plus exige une mise en place très rigoureuse; le grand malheur a été provoqué par la charge de glace qui se formait sur la face aval de la vanne en ouverture partielle et qui parfois la recouvrait complètement, atteignant par endroit plusieurs mètres d'épaisseur.

Les quelques essais que l'on a effectués sur des ouvrages équipés de cette vanne-secteur montrent que c'est vraiment inquiétant en ouverture d'urgence en hiver ou en ouverture précoce au printemps alors que la nuit la température descend au-dessous du point de congélation de l'eau; il s'accumule alors beaucoup de glace sur la vanne et les mécanismes de levage. C'est ce risque qui nous a fait rejeter les vannes-secteurs.

Quant aux vannes planes qui équipent nos évacuateurs, la moitié d'entre elles sont chauffées et à double paroi; un système de chauffage électrique est installé à l'intérieur des parois amont et aval pour dégager complètement la glace qui peut se former à la face aval.

M. ROBERT. - Bien que la question sorte du thème du colloque, puis-je demander à M. MARINIER de dire quelques mots du transport de l'électricité depuis les complexes de production jusqu'aux lieux d'utilisation dans le Sud?

$\mathrm{Par}$ leurs rapports aux dernières Conférences Internationales des Grands Réseaux Electriques, les ingénieurs québecois ont montré qu'ils avaient fait de très belles choses aussi dans ce domaine.

M. MARINIER. - Pour le transport de l'énergie depuis la Baie James jusqu'à Montréal (1000 km environ), il y a eu, au début des années 1970 , un réexamen de ce qui avait été fait précédemment. On s'est alors interrogé sur des formules autres telles que le transport en courant continu ou à une tension supérieure à $1000 \mathrm{kV}$. Le verdict, à l'époque, a été de ne pas prendre de risques et d'utiliser une technologie maîtrisée, tant chez le fournisseur que chez le constructeur. On a done répété l'expérience de Churchill Falls et de Manicouagan et c'est la raison pour laquelle vous avez pu voir dans le film que toute 
l'énergie du Complexe La Grande Phase I, soit plus de $10000 \mathrm{MW}$, est transportée par un réseau à $735 \mathrm{KV}$, je ne sais pas si pour les autres complexes que La Grande, la même solution sera retenue mais pour le moment, c'est par raison de sécurité que le système à $735 \mathrm{kV}$ a été adopté

$\mathrm{Au}$ cours du développement du complexe de La Grande par deux fois on a modifié le type des tours : dans un premier temps, il y a eu les tours autoportantes classiques, puis les tours de type haubanné en $V$ avec deux mâts et une traverse, qui ne sont vraiment plus des tours, et enfin le dernier type à chaînette qui est haubanné et qui ressemble au précédent mais dans lequel la traverse en treillis métallique est remplacée par un câble reliant le sommet des deux mâts. Ce dernier type déroute un peu les nouveaux venus qui se demandent comment tient cet ensemble.

M. BARRIER. - Ne pensez-vous pas que la création de toutes ces retenues risque de modifier l'hydrologie? D'autre part, quels espoirs peuvent avoir les entreprises françaises quant à leur participation à la réalisation des aménagements en projet que vous venez de nous présenter.

M. MARINIER. - Dans notre cas, la formation des grands réservoirs a été l'obstacle à surmonter dans le début des année 1970. Les ingénieurs étaient fiers de construire de grandes retenues mais les journaux se sont lancés à la défense des autochtones. Les retenues feront passer la surface d'eau du territoire de 3 à $5 \%$. Il y a déjà beaucoup de surfaces d'eau sur ce territoire, c'est commun dans la Taïga ; il y a déjà des milliers de lacs et il n'est pas tellement dramatique que le rapport de surface inondée passe de 3 à $5 \%$. A ceux qui clament qu'on va noyer les territoires de chasse, on pourrait répondre que par contre on va agrandir les territoires de pêche. çaise?

$M$. le Président. - Y a-t-il un espoir pour l'industrie fran-

M. MARINIER. - Ce sont surtout les grandes machines qui sont exportables. On trouve sur place les matériaux de construction; les engins de construction sont américains et souven normalisés de sorte qu'il est facile d'obtenir les pièces de rechange. En ce qui concerne l'électromécanique, deux sociétés ont déjà 10 ans et plus d'expérience, notamment Marine Industrie, qui est une filiale du groupe Alsthom, et les Ateliers d'Ingénierie Dominion, qui sont une filiale du Groupe General Electric. Ces maisons d'origine québecoise sont en activité depuis le début des années 1930. Tout dernièrement, s'est installé la firme Sulzer en association avec un fabricant montréalais. Il semble que ce nouveau venu a bien l'intention de partager les 67 machines qui restent à fournir d'ici la fin du siècle.

Je ne dis pas qu'il n'y a pas de place pour d'autres constructeurs mais il y a une nette préférence pour les ateliers du Québec.

M. COTILLON. - Une question me "démange" : je veux parler des moustiques. On dit qu'à Bratsk le moustique est responsable d'une chute de la productivite de $30 \%$. Je voudrai savoir quels ennuis vous avez eux avec les moustiques et comment vous $\mathrm{y}$ faites face.

M. MARINIER. - Les moustiques, c'est terrible. Heureusement, sur nos chantiers, on peut maîtriser ce fléau par des arrosages aériens des zones de reproduction et des lieux de travail. C'est une technique qui a été mise au point et qui est peu coûteuse; on arrose régulièrement les chantiers du mois de juin au mois d'août, ainsi les gens des campements et villages ne sont pas incommodés, ni les conducteurs d'engins sur les ouvrages où il y a suffisamment d'activité

Les moustiques les indisposent quand ils vont à la pêche et cela gâche complètement leur plaisir ; on a beau mettre des vestes insectifuges ou s'enduire de toutes sortes de lotions, cela n'y fait pas. Quand on discute avec les gens qui y habitent, ils nous disent : vivement l'hiver car c'est tellement mieux partir du moment où il fait froid, on peut alors sortir. L'hiver est très clair, très lumineux. La bonne saison va de février à avril. L'été, c'est moins plaisant, il fait plus chaud mais le temps est relativement pluvieux et il y a toujours ces bestioles dès que l'on s'éloigne des habitations ou des chantiers.

M. le Président. - Les impératifs de l'horaire nous obligent à passer maintenant à la projection du deuxième film, et ce sera très probablement la fin de la séance.

(Projection du deuxième film). 


\section{Abstract}

\section{Hydro-electric development of James bay}

The James Bay Hydro Company has been mandated by the Government of Quebec to develop the waterways in the territory of James Bay to meet electricity requirements in the 1980 's. The territory has a total area of $176000 \mathrm{~km}^{2}$, i.e. close to one-third of the area of France.

Whereas earlier projects, the Churchill and the Maniconagan - Outardes heads, (had been developed quite near the main communication routes, those in James Bay were developed in a quasi-Siberian environment where it was necessary to construct the transport and accommodation inf rastructure.

Atter ten years of work, over $85 \%$ of the initial phase of the La Grande Rivière hydro-electric plant has now been completed. However, the development of the territory's rivers is far from complete. Twenty years will be needed to complete the second phase of the complex and implement two other projects: the Grande Rivière de la Baleine plant and the Nottaway, Broadback and Rupert river complex.

The initial phase of the $\mathrm{La}$ Grande Complex - as it is known - includes the construction of three power stations, La Grande 2, La Grande 3 and La Grande 4 on the La Grande river. This river drains a $98000 \mathrm{~km}^{2}$ basin with an average flow of $1700 \mathrm{~m}^{3} / \mathrm{sec}$.

The underground station of La Grande 2, whose sixteen turbo-generator sets produce a total installed power of $5328 \mathrm{MW}$, has been in operation since 1981. The dam (length at the crest: $2835 \mathrm{~m}$, height $160 \mathrm{~m}$ ), has necessitated a 23 million $\mathrm{m}^{3}$ of earth fill, i.e. the same volume as was required for the thirty dykes retaining the water of the $2835 \mathrm{~km}^{2}$ reservoir. The spillway can discharge $15300 \mathrm{~m}^{3} / \mathrm{sec}$.

The La Grande 3 station is equipped with twelve Francis turbines driving the same number of generators, which will produce 12.3 billion $\mathrm{kWh}$. The first set was commissioned in June of last year. The La Grande 3 dam, the longest of the complex was completed in October 1980. The construction required a total volume of rockfill of 22.2 million $\mathrm{m}^{3}$. The
$2460 \mathrm{~km}^{2}$ reservoir is confined by 67 dykes. The spillway is designed to discharge $10100 \mathrm{~m}^{3} / \mathrm{sec}$.

The La Grande 4 station, like La Grande 3, is overground. With an installed power of $2637 \mathrm{MW}$, it will operate at full load in 1985, yielding an annual output of 14.1 billion $\mathrm{kWh}$. Construction of the dam was completed in 1981:3600 m long, $125 \mathrm{~m}$ high, 20 million $\mathrm{m}^{3}$ of earthtill. There are eight dykes which correspond to an additional volume of 14 million $\mathrm{m}^{3}$ of till.

Two by-passes have been excavated to increase the potential of the La Grande river through two make-up reservoirs: the Eastmain by-pass and the Caniapiscau by-pass.

The second phase of the $\mathrm{La}$ Grande plant includes the construction of five power stations, La Grande 1, Brisay, Laforge 1, Laforge 2 and Eastmain 1, which will add over 3200 MW to Quebec Hydro's installed power.

The La Grande-Baleine plant will be installed on the Grande rivière de la Baleine, which is approximately at the same latitude as Moscow. Under the scheme construction is planned of three power stations (Grande-Baleine 1, Grande-Baleine 2 and Grande-Baleine 3), three spillways, one regulating structure and three new reservoirs. The total installed power of this plant will be $2900 \mathrm{MW}$. Under the programme presented by Quebec Hydro to the Government the first turbines would enter into service by 1990 .

The catchment basins of the three rivers, the Nottaway, the Broadback and the Rupert, where another complex (the NBR) will be developed, are located approximately $300 \mathrm{~km}$ south of $\mathrm{La}$ Grande Riviere. By combining the flows of these three rivers via relatively short canals and installing the bulk of the equipment on the lower section of the Broadback, it will be possible to obtain an annual output of approximately 47 billion $\mathrm{kWh}$, i.e. approximately two-thirds of the output of the initial phase of the La Grande plant. Eleven power stations will be built, with a total installed power of $7255 \mathrm{MW}$. 